\title{
Trends Richtung
}

\section{Akademisierung der Psychotherapie-Weiterbildung}

Peter Schulthess

Der Trend zur Akademisierung der Psychotherapie-Weiterbildung ist unübersehbar. Lange Zeit verstanden sich die Universitäten als Ort der wissenschaftlichen Ausbildung, nicht der Berufsausbildung. Die Psychotherapieausbildung als Berufsausbildung fand entweder parallel oder nachfolgend an privaten Ausbildungsinstituten statt. Das hat sich auch in der Schweiz sehr verändert. Praktisch alle Universitäten bieten heute eigene postgraduale Weiterbildungsgänge in Psychotherapie an, zumeist in Verhaltenstherapie. Es sind jedoch auch andere Richtungen auffindbar, je nach Präferenz der entsprechenden LehrstuhlinhaberInnen. In der Regel werden diese Studiengänge in Partnerschaft mit einem bestehenden privaten Institut realisiert.

Die Vorteile liegen auf der Hand: Anschluss an Forschung und wissenschaftliche Therapietheorievermittlung, Abschluss mit einem akademischen Grad (MAS) als akademische Zusatzqualifikation nach dem MSc oder Doktorat.

Was bedeutet das für die nicht an eine Universität angeschlossenen privaten Weiterbildungsgänge in der Schweiz? Der Druck im Zuge der Akkreditierungsverfahren nach vermehrter Forschung und wissenschaftlicher Evaluation ist unverkennbar und hat bei vielen $\mathrm{zu}$ entsprechenden Auflagen geführt. Forschung kann jedoch nur in grösserem Verbund gemacht werden, zumindest empirische Forschung. Letztere ist zwingend, solange das Prinzip der Evidenzbasierung nach dem medizinischen Modell der «evidence based medicine» auch in der Psychotherapie gilt, um als Verfahren anerkannt zu bleiben. Wie können sich die privaten Institute da positionieren? Haben sie auf längere Frist einen Wettbewerbsnachteil? Was für andere Kooperationen als die Partnerschaft in einem MAS-Weiterbildungsgang sind denkbar, um Zugang zu Forschungsgeldern und Forschungs-Know-how zu erhalten, wie auch zur Vermittlung von evidenzbasiertem Grundlagenwissen auf dem sich so rasch entwickelnden Forschungsgebiet der Psychotherapie? Ist über kurz oder lang die Vielfalt der Psychotherapie in der Schweiz bedroht? In der ersten Akkreditierungsrunde sind bereits einige Therapierichtungen ausgeschieden, auch solche mit wissenschaftlicher Anerkennung in anderen Ländern.

Das Psychologieberufegesetz (PsyG) hat die Trennung zwischen Ausbildung (Psychologie-
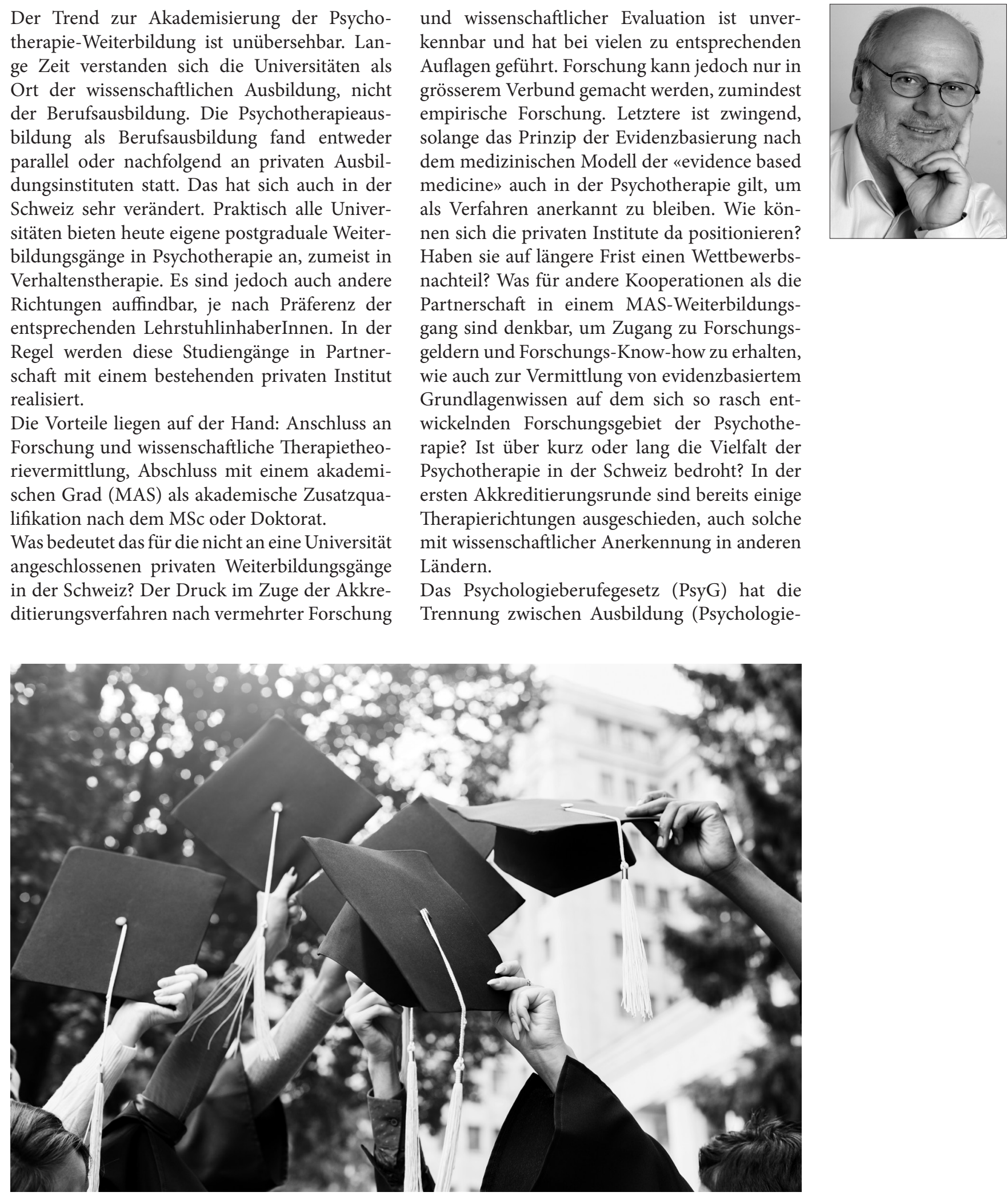
studium) und Weiterbildung verfestigt. Überschneidungen zwischen Psychologiestudium und Psychotherapieweiterbildung sind geächtet und dürfen nicht stattfinden, auch wo das in Einzelfällen als unverständlich und fachlich nicht gerechtfertigt angesehen werden muss. Diese strikte Trennung steht im Gegensatz zu einem Trend, der in anderen europäischen Ländern im Aufwind ist. So wird seit Jahren an der Sigmund Freud PrivatUuniversität Wien (SFU) und an einigen Schwesteruniversitäten in anderen Ländern ein Studiengang in Psychotherapiewissenschaft angeboten, der wissenschaftliches Grundlagenwissen und Therapieausbildung sowie klinische Praxis vereint, ganz im Sinne einer wissenschaftlichen Berufsausbildung zum Psychotherapeuten. In Deutschland wurde vor Kurzem eine Revision des Psychotherapiegesetzes verabschiedet, die Direktstudiengänge in Psychotherapie vorsieht. Die Trennung zwischen Grundstudium in Psychologie und Weiterbildung in Psychotherapie wird damit aufgehoben, ausgerechnet in Deutschland, nach dessen Modell die Schweiz ihr PsyG ausgerichtet hat. Man wird künftig direkt ab Matura ein Studium der Psychotherapie machen können, das mit dem Abschluss auch eine Praxisberechtigung nach sich zieht. Wie lange wird es dauern, bis das PsyG entsprechend revidiert wird, um solche Studiengänge auch an Schweizer Hochschulen anbieten zu können? Was wird das für die privaten Institute bedeuten?

Nun, die Schweiz braucht in der Regel etwa zehn Jahre Zeit, bis sie dem deutschen Modell nachfolgt. Dennoch sollte die Diskussion darüber schon heute stattfinden. Was sind die Vorteile eines solchen Direktstudienganges? Was sind seine Nachteile? Wie weit wird damit die Vielfalt der Psychotherapierichtungen gefährdet? Was für Kooperationen privater Institute mit Hochschulen sind denkbar? Wie sehen erste Erfahrungen aus?

Um solchen Fragen vertieft nachzugehen, veranstaltet die ASP am 28. März eine Tagung zum
Thema «Trends Richtung Akademisierung der Psychotherapie-Weiterbildung».

Es werden zwei Modelle von existierenden Partnerschaften privater Institute mit Hochschulen vorgestellt, die seit einigen Jahren einen akkreditierten Weiterbildungsgang mit MAS-Abschluss anbieten: «Systemisch-verhaltenstherapeutische Psychotherapie» an der ZHAW und «Personzentrierte Psychotherapie» an der Universität Basel. Die VertreterInnen der Hochschulen, Prof. Imke Knafla und Prof. Jens Gaab, und der privaten Partnerinstitute, Rainer Bürki und Julia Besewski, werden Auskunft geben über die Gründe, warum sie diese Verbindung eingegangen sind und was aus ihrer jeweiligen Sicht und Erfahrung die Vorteile, aber auch etwaige Probleme sind.

Von der SFU wird die Leiterin des Studienganges Psychotherapiewissenschaft, Prof. Jutta Fiegl, berichten, wie die Zusammenarbeit mit privaten Instituten im Rahmen dieses Direktstudienganges aussieht und was für Erfahrungen damit gemacht werden. Aus Deutschland orientiert uns ein Mitglied des Vorstandes der Bundespsychotherapeutenkammer, Nikolaus Melkop, über die Reform des Psychotherapeutengesetzes und was von den Direktstudiengängen zu erwarten oder befürchten ist. Aus Sicht der Universität Zürich wird sich Prof. Christoph Flückiger zum Thema äussern.

Verpassen Sie nicht die Gelegenheit, sich aus erster Hand über diese Themen orientieren zu lassen und am Diskurs teilzunehmen!

Peter Schulthess ist Vorstandsmitglied der ASP.

ASP-Tagung in Zürich, Samstag 28. März 2020

Thema: «Trends Richtung Akademisierung der Psychotherapie-Weiterbildung»"

Referent*innen: Prof. Imke Knafla (ZHAW), Prof. Jens Gaab (Uni Basel), Prof. Jutta Fiegl (SFU Wien), Prof. Christoph Flückiger (Uni Zürich), Nikolaus Melkop (Bundespsychotherapeutenkammer), Rainer Bürki (pca), Julia Besewski (ZSB) 